\title{
3D measurement of human face by stereophotogrammetry
}

\author{
Holger Wagner, Axel Wiegmann, Richard Kowarschik, Friedrich Zöllner \\ Friedrich-Schiller-University Jena, Institute of Applied Optics \\ Froebelstieg 1, 07743 Jena, Germany \\ mailto: holger.wagner@uni-jena.de
}

\begin{abstract}
In this article a rapid and self-calibrating stereophotogrammetry based method for 3D measurements is described. The approach uses a series of statistical generated illumination patterns to encode the surface under test. The allocation of homologous points, which is necessary for 3D reconstruction by triangulation, is done by an adapted correlation technique.
\end{abstract}

\section{Introduction}

The advantages of optical measurements like fast data acquisition, non-interacting with the object under test or the possibility of soft tissue measurements were used in a wide range of technical and medical applications [1-6].

The background of our activities was to adapt a method for function orientated diagnostics and therapy in dentistry to provide prognoses for jawgrowth or surgical procedures.

The aim was to get a dense 3D point cloud of the human face by a single, rapid measurement. The two named requirements were posted to avoid measurement errors caused by movements of the person's head and to overcome difficulties of matching point clouds effected by inevitable changing's in facial expressions.

\section{Adapted photogrammetrical method}

The adapted photogrammetrical method consists of four essential components.

At first, for image acquisition and providing of coordinates for $3 \mathrm{D}$ reconstruction by triangulation a convergent arrangement of two cameras is used. Actually, the cameras are calibrated by a previous calibration procedure to get necessary parameters like focal length, main point coordinates and those which describe deviations, i.e. distortion or shear, from an idealized pinhole model.

Furthermore, for encoding the object shape a special illumination is adapted which maps a sequence of about 20 statistically generated patterns onto the object (see figure 1). Therefore, every point of the object surface is characterized by an individual sequence of intensity values. This approach makes it possible to use a single sensor element for allocating homologous points. It is not necessary to take a spatial template, i.e. nine or eleven pixels in square, into account. As a result, especially at higher profile gradients a denser point cloud is generated.

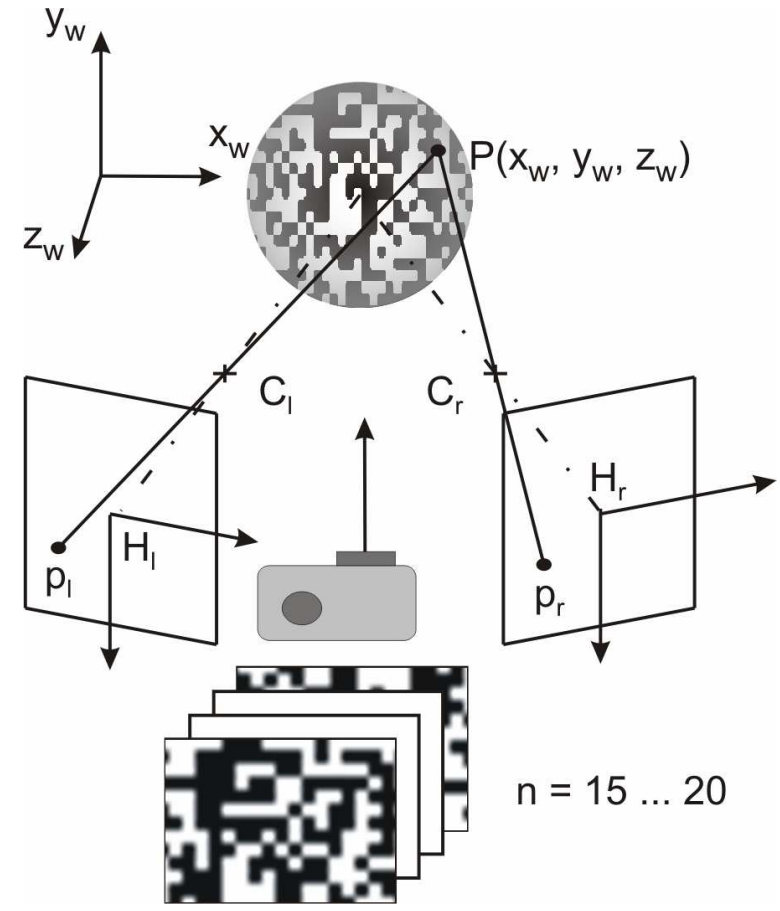

Fig. 1 Schematic arrangement of measurement setup (two cameras and projector, including image sequence)

The third main component is a correlation technique for allocating homologous points. The correlation coefficients of each point of the first camera and those, which are in relating areas of the second camera, are computed. A point is accepted as a homologous one if the maximum of the correlation coefficients exceeds a certain threshold.

At least a self calibration of the extrinsic parameters is implemented. This feature makes the arrangement insensitive against environmental changes because the geometric relations of the cameras are determined at the period of measurement. The arrangement has to be time stable only while the period of image capturing. This approach is possible because every pair of homologous points provides four coordinates for the determination of the three unknown values of the 3D point. 
Consequently, if a sufficient number of homologous points is known, it is possible to determine the 3D points and the extrinsic parameters.

\section{Experimental setup}

The previously described photogrammetrical method is realized by an experimental setup, which is shown in figure 2. Note that it is not necessary to use a digital projector like it is pictured in figure 2. Also a classical one or the usage of more than a single direction of illumination, i.e. to prevent not or low illuminated areas, is possible.

The spatial resolution, caused by camera resolution and reproduction scale, is about $0.2 \mathrm{~mm}$ whereas the longitudinal resolution covers a range of 0.4 to $0.8 \mathrm{~mm}$ depending on the angle between the cameras.

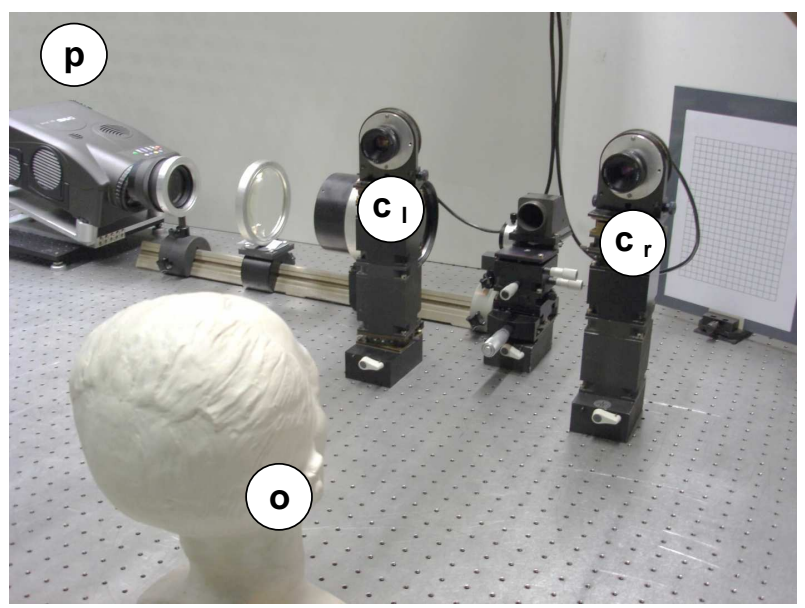

Fig. 2 Experimental setup with projector (p), left and right camera (cl, cr)and $3 D$ object (o)

\section{Results of 3D measurements}

Figure 3 shows two examples of reconstructed 3D objects. The measured human face consists of about 200,000 points and is pictured with rendering algorithms of OpenGL. Areas with a lower density of measured points are caused by low reflectance, e.g., at eyebrows or by masking of the surface.

The right part of figure 3 shows a point cloud of a shuttlecock and clarifies that also technical objects with complex structures can be measured.

For experimental tests regarding of precision and uncertainty of measurement we used a matt finished aspherical lens. The deviations from the reference are determined in a range of $+/-0.2 \mathrm{~mm}$ whereas the rms error is about $0.1 \mathrm{~mm}$.

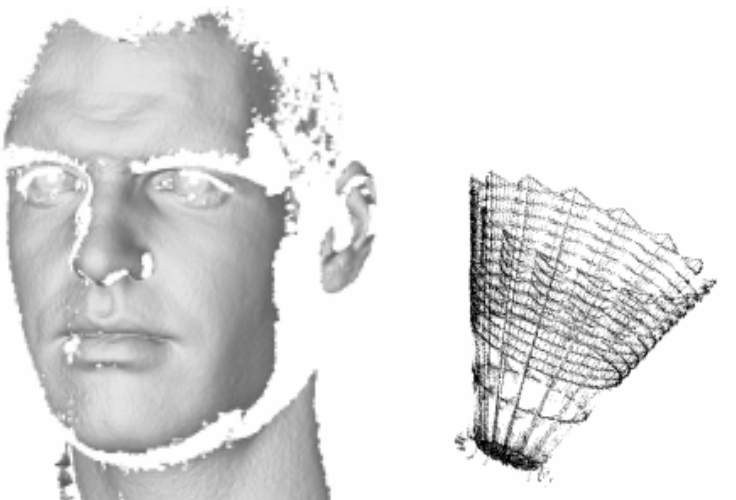

Fig. 3 Examples of measured $3 D$ objects: Human face and a shuttlecock

\section{Conclusions}

In this work a possibility of a rapid 3D measurement by stereophotogrammetry is shown. In addition to the low measurement period $(<1$ second), which is determined by the digital cameras, a dense point cloud is generated. The accuracy of about $+/-0.1 \mathrm{~mm}$ (rms) is sufficient for a wide range of medical or technical applications.

\section{Acknowledgements}

This project was supported by the Thuringia ministry of science, research and culture under the topic: '3D shape measurement for function orientated diagnostic and therapy in dentistry'.

\section{References}

[1] W. Schreiber, G. Notni: Opt. Eng. 39, pp. 159-169 (2000)

[2] R. Kowarschik, P. Kühmstedt, J. Gerber, W. Schreiber, G. Notni: Opt. Eng. 39, pp. 150-158 (2000)

[3] F. Zöllner, V. Matusevich, R. Kowarschik: Proc. of SPIE Vol. 5144 (2003)

[4] P. Albrecht, B. Michaelis: Proc. 14th Int. Conf. on Pattern Recognition, Volume I, Brisbane, Australia, pp. 845-849 (1998)

[5] M. Kujawinska, L. Salbut, K. Patorski: Appl. Opt. 30, 1633 (1991)

[6] G. Sansoni, M. Carocci, S. Lazzari, R. Rodella J. Opt. A: Pure Appl. Opt. 1, 83 (1999) 